\title{
The Exact Solution of the Space-Time Fractional Modified Kdv-Zakharov-Kuznetsov Equation
}

\author{
Qiuyan Jin1, Tiecheng Xia1,2*, Jinbo Wang² \\ ${ }^{1}$ Department of Mathematics, Shanghai University, Shanghai, China \\ ${ }^{2}$ Science and Technology on Communication Security Laboratory, Chengdu, China \\ Email: qiuyanjin815@163.com, ^xiatc@shu.edu.cn, doctor03@163.com
}

How to cite this paper: Jin, Q.Y., Xia, T.C. and Wang, J.B. (2017) The Exact Solution of the Space-Time Fractional Modified KdvZakharov-Kuznetsov Equation. Journal of Applied Mathematics and Physics, 5, 844852.

https://doi.org/10.4236/jamp.2017.54074

Received: March 3, 2017

Accepted: April 25, 2017

Published: April 28, 2017

Copyright $\odot 2017$ by authors and Scientific Research Publishing Inc. This work is licensed under the Creative Commons Attribution International License (CC BY 4.0).

http://creativecommons.org/licenses/by/4.0/

\begin{abstract}
In this paper, we get many new analytical solutions of the space-time nonlinear fractional modified KDV-Zakharov Kuznetsov (mKDV-ZK) equation by means of a new approach namely method of undetermined coefficients based on a fractional complex transform. These solutions have physics meanings in natural sciences. This method can be used to other nonlinear fractional differential equations.
\end{abstract}

\section{Keywords}

Analytical Solutions, the Space-Time Fractional Modified KDV-ZK

Equation,Nonlinear Fractional Differential Equation, Modified

Riemann-Liouville Derivative

\section{Introduction}

Nonlinear fractional differential equations (NFDEs) are universal models of the classical differential equations of integer order. In recent years, the fractional order derivative and integral is becoming a hot spot of international research; it can more accurately describe the nonlinear phenomena in physics. Such as chemical kinematics, chemical physics and geochemistry, communication, physics, biology, engineering, mathematics, diffusion processes in porous media, in vibrations in a nonlinear string, power-law non-locality, and power-law longterm memory can use NFDEs as models to express these problem [1] [2] [3] [4] [5]. In the last few years, it has become an important issue and matter of interest for researchers about the study of analytical and numerical solutions of fractional differential equations (FDEs). There are a lot of effective methods which can be used to study soliton, such as the fractional functional sub-equation method [6], the fractional modified trial equation method [7], the first integral method 
[8], the fractional functional variable method [9], the extended tanh-function method [10], the (G'G)-expansion method [11] [12] and so on.

The present article aims to find out the modified KDV-Zakharov Kuznetsov [13] [14] [15] equation's exact solutions by using named method of undetermined coefficients. The following is the organization of this paper. Some basic definitions and mathematical preliminaries of the fractional calculus are introduced in the next section. Investigated method of undetermined coefficients applied to solve fractional differential equations based on a fractional complex transform is presented in Section 3. In Section 4, we apply method of undetermined coefficients to the space-time nonlinear fractional modified KDV-ZK equation. Finally, we give some conclusions.

\section{Basic Definitions}

Fractional calculus is a generalization of classical calculus. There are a lot of approaches developed over years to generalize the concept of fractional order derivative, such as, Riemann-Liouville, Grünwald-Letnikow, Caputo [16], KolwankarGangal, Oldham and Spanier, Miller and Ross, Cresson have presented many methods, and Jumnarie put forward a modified Riemann-Liouville derivative [17] [18].

In the section, the some properties and definitions of the modified RiemannLiouville derivative that will be applied in the sequel of the work were given.

The following is the modified Riemann-Liouville derivative defined by Jumarie [17] [18]

$$
D_{t}^{\alpha} f(t)=\left\{\begin{array}{l}
\frac{1}{\Gamma(-\alpha)} \int_{0}^{t}(t-\xi)^{-\alpha-1}[f(\xi)-f(0)] \mathrm{d} \xi, \alpha<0, \\
\frac{1}{\Gamma(1-\alpha)} \frac{\mathrm{d}}{\mathrm{d} t} \int_{0}^{t}(t-\xi)^{-\alpha}[f(\xi)-f(0)] \mathrm{d} \xi, 0<\alpha<1, \\
\left(f^{(n)}(t)\right)^{(\alpha-n)}, n \leq \alpha \leq n+1, n \geq 1 .
\end{array}\right.
$$

Remark1. $f: R \rightarrow R, t \rightarrow f(t)$ denote a continuous but not necessarily differentiable function.

The probability calculus, fractional Laplace problems, and fractional variational calculus successfully applied Jumarie's modified Riemann-Liouville derivative. To summarize a few useful formulae by Jumarie's modified RiemannLiouville derivative in [17] [18], we give some properties as follows

$$
\begin{gathered}
D_{t}^{\alpha} t^{\gamma}=\frac{\Gamma(\gamma+1)}{\Gamma(\gamma+1-\alpha)} t^{\gamma-\alpha}, \quad \gamma>0, \\
D_{t}^{\alpha}(c f(t))=c D_{t}^{\alpha} f(t), \quad c=\text { constant, } \\
D_{t}^{\alpha} f[g(t)]=f_{g}^{\prime}[g(t)] D_{t}^{\alpha} g(t), \\
D_{t}^{\alpha} f[g(t)]=D_{g}^{\alpha} f[g(t)]\left(g^{\prime}\right)^{\alpha},
\end{gathered}
$$




$$
D_{t}^{\alpha}[a f(t)+b g(t)]=a D_{t}^{\alpha} f(t)+b D_{t}^{\alpha} g(t) .
$$

Remark 2. J. H. He et al. in [19] modified the chain rule given by Equation (5) to the formula

$$
D_{t}^{\alpha} f[g(t)]=\sigma_{t}^{\prime} f_{g}^{\prime}[g(t)] D_{t}^{\alpha} g(t),
$$

where $\sigma_{t}^{\prime}$ is called the sigma indexes (see [19]). Therefore, Equation (5) is modified to the forms

$$
D_{t}^{\alpha} f[g(t)]=\sigma_{t}^{\prime} D_{g}^{\alpha} f[g(t)]\left(g^{\prime}\right)^{\alpha}
$$

\section{Method of Undetermined Coefficients}

In the section, we introduce the generally steps of method of undetermined coefficients

Step 1: We set a nonlinear fractional order partial differential equation as follows

$$
P\left(u, D_{t}^{\alpha} u, D_{x}^{\beta} u, D_{t}^{\alpha} D_{t}^{\alpha} u, D_{t}^{\alpha} D_{x}^{\beta} u, D_{x}^{\beta} D_{x}^{\beta} u, \cdots\right)=0,0<\alpha, \beta<1
$$

where $u$ is an unknown function about $x, t$ two independent variables, $D_{t}^{\alpha} u, D_{x}^{\alpha} u$ modified Riemann-Liouville derivative of $u$, and $P$ is a polynomial of $u$ and its partial fractional derivatives, in which includes the highest order derivatives and the nonlinear terms.

Step 2: By using the traveling wave transformation

$$
\begin{aligned}
& u(x, t)=U(\xi), \\
& \xi=\frac{k x^{\beta}}{\Gamma(\beta+1)}-\frac{c t^{\alpha}}{\Gamma(\alpha+1)},
\end{aligned}
$$

where $k$ and $c$ are non zero arbitrary constants. And by using the chain rule

$$
\begin{aligned}
& D_{t}^{\alpha} u=\sigma_{t}^{\prime} \frac{\mathrm{d} U}{\mathrm{~d} \xi} D_{t}^{\alpha} \xi, \\
& D_{x}^{\alpha} u=\sigma_{x}^{\prime} \frac{\mathrm{d} U}{\mathrm{~d} \xi} D_{x}^{\alpha} \xi,
\end{aligned}
$$

where $\sigma_{t}^{\prime}$ and $\sigma_{x}^{\prime}$ are called the sigma index. The sigma index usually is determined by gamma function [20]. In general, we can take $\sigma_{t}^{\prime}=\sigma_{x}^{\prime}=l$, where $l$ is a constant.

Substituting (10) along with (2) and (11) into (9), we can rewrite Equation (9) in the following nonlinear ordinary differential equation

$$
Q\left(U, U^{\prime}, U^{\prime \prime}, U^{\prime \prime \prime}, \cdots\right)=0,
$$

where the prime denotes the derivative with respect to $\xi$. For the convenience of calculation, we should obtain a new equation by integrating Equation (12) term by term one or more times.

Step 3: By the following form [21], assume that solution of the Equation (14) can be represented

$$
U(\xi)=\operatorname{Asech}^{m} \xi,
$$


where $A$ is nonzero constant, $m$ is obtained by balancing the highest order term and nonlinear term of Equation (9) or Equation (12).

Step 4: Substituting the constant $A$ and $m$ into Equation (14), we can obtain the solution of the fractional order Equation (9).

\section{The $(3+1)$ Dimensional Space-Time Fractional mKDV-ZK Equation}

In this current sub-section, we apply method of undetermined coefficients to solve the $(3+1)$ dimensional space-time fractional mKDV-ZK equation of the form,

$$
D_{t}^{\alpha} u+\mathrm{d} u^{2} u_{x}+e u_{x x x}+f u_{x y y}+g u_{x z z}=0, \quad t>0,0<\alpha<1,
$$

where $d, e, f$ and $g$ are nonzero constants, $\alpha$ is a parameter describing the order of the fractional space-time-derivative. When $f=0, g=0, d$, $e \neq 0$, Equation (14) is called the fractional modified KDV equation

$$
D_{t}^{\alpha} u+\mathrm{d} u^{2} u_{x}+e u_{x x x}=0, \quad t>0,0<\alpha<1,
$$

when $\alpha=1$, Equation (14) is called the modified KDV-ZK equation

$$
u_{t}+\mathrm{d} u^{2} u_{x}+e u_{x x x}+f u_{x y y}+g u_{x z z}=0, \quad t>0 .
$$

The modified KDV-ZK equation is applied in many physical areas. Existence of the solutions for this equation has been considered in several papers, see references in [22] [23]. Next, we will obtain the non-topological soliton and dark soliton solutions to Equation (14) by method of undetermined coefficients [24] [25].

Therefore, we use the following transformations,

$$
u(x, y, z, t)=U(\xi), \quad \xi=k x+p y+q z-\frac{\lambda t^{\alpha}}{\Gamma(1+\alpha)},
$$

Where $k, p, q, \lambda$ are nonzero constants.

Substituting Equation (17) with Equation (2) and Equation (11) into Equation (14), we have

$$
-\lambda U^{\prime}+k d U^{2} U^{\prime}+k^{3} e U^{\prime \prime \prime}+k f p^{2} U^{\prime \prime \prime}+k g q^{2} U^{\prime \prime \prime}=0,
$$

where " $U$ "” $=\frac{\mathrm{d} U}{\mathrm{~d} \xi}$. By once integrating and setting the constants of integration to zero, we obtain

$$
-\lambda U+\frac{k d}{3} U^{3}+k\left(e k^{2}+f p^{2}+g q^{2}\right) U^{\prime \prime}=0 .
$$

\subsection{The Non-Topological Soliton Solution}

To get the non-topological soliton solution of Equation (19), we can make the assumption,

$$
U(\xi)=\operatorname{Asech}^{m} \xi
$$


where

$$
\xi=k x+p y+q z-\frac{\lambda t^{\alpha}}{\Gamma(1+\alpha)},
$$

where $k, p, q, \lambda$ are nonzero constants coefficients. The $m$ is unknown at this point and will be determined later. From the Equation (20)-(21), we obtain

$$
\frac{\mathrm{d} U(\xi)}{\mathrm{d} \xi}=-A m \operatorname{sech}^{m+1} \xi \sinh \xi,
$$

and

$$
\begin{aligned}
\frac{\mathrm{d}^{2} U(\xi)}{\mathrm{d} \xi^{2}} & =-A m(m+1) \operatorname{sech}^{m} \xi(-\operatorname{sech} \xi \tanh \xi) \sinh \xi+\left(-A m \operatorname{sech}^{m+1} \xi \cosh \xi\right) \\
& =A m(m+1) \operatorname{sech}^{m+2} \xi \sinh ^{2} \xi-A m \operatorname{sech}^{m} \xi \\
& =A m(m+1) \operatorname{sech}^{m+2} \xi\left(\frac{1}{\operatorname{sech}^{2} \xi}-1\right)-A m \operatorname{sech}^{m} \xi \\
& =A m^{2} \operatorname{sech}^{m} \xi-A m(m+1) \operatorname{sech}^{m+2} \xi
\end{aligned}
$$

and

$$
U^{3}(\xi)=A^{3} \operatorname{sech}^{3 m} \xi .
$$

Thus, substituting the ansatz (23)-(27) into Equation (21), yields to

$$
\begin{aligned}
& -\lambda A \operatorname{sech}^{m} \xi+\frac{k d}{3} A^{3} \operatorname{sech}^{3 m} \xi \\
& +k\left(e k^{2}+f p^{2}+g q^{2}\right)\left(A m^{2} \operatorname{sech}^{m} \xi-A m(m+1) \operatorname{sech}^{m+2} \xi\right)=0 .
\end{aligned}
$$

Now, from Equation (25), equating the exponents $m+2$ and $3 m$ leads to

$$
m+2=3 m,
$$

so that

$$
m=1 .
$$

From Equation (25), setting the coefficients of $\operatorname{sech}^{m+2} \xi$ and $\operatorname{sech}^{3 m} \xi$ terms to zero, we obtain

$$
\frac{k d}{3} A^{3}-A k\left(e k^{2}+f p^{2}+g q^{2}\right) m(m+1)=0,
$$

by using Equation (27) and after some calculations, we have

$$
A= \pm \sqrt{\frac{6\left(e k^{2}+f p^{2}+g q^{2}\right)}{d} .}
$$

We find, from setting the coefficients of $\operatorname{sech}^{m} \xi$ terms in Equation (25) to zero

$$
-\lambda A+A m^{2} k\left(e k^{2}+f p^{2}+g q^{2}\right)=0,
$$

also we get

$$
\lambda=k\left(e k^{2}+f p^{2}+g q^{2}\right) .
$$


From Equation (29), it is important to note that

$$
d\left(e k^{2}+f p^{2}+g q^{2}\right)>0 .
$$

Thus finally, the 1-soliton solution of Equation (14) is given by:

$$
\begin{gathered}
u_{1}(x, y, z, t)=\sqrt{\frac{6\left(e k^{2}+f p^{2}+g q^{2}\right)}{d}} \operatorname{sech}\left(k x+p y+q z-\frac{d\left(e k^{2}+f p^{2}+g q^{2}\right) t^{\alpha}}{\Gamma(1+\alpha)}\right), \\
u_{2}(x, y, z, t)=-\sqrt{\frac{6\left(e k^{2}+f p^{2}+g q^{2}\right)}{d}} \operatorname{sech}\left(k x+p y+q z-\frac{d\left(e k^{2}+f p^{2}+g q^{2}\right) t^{\alpha}}{\Gamma(1+\alpha)}\right) .
\end{gathered}
$$

\subsection{The Dark Soliton Solution}

In order to start off with the solution hypothesis, we use the solitary wave ansatz of the form

$$
U(\xi)=A \tanh ^{m} \xi,
$$

and

$$
\xi=k x+p y+q z-\frac{\lambda t^{\alpha}}{\Gamma(1+\alpha)},
$$

where $k, p, q, \lambda$ are the free parameters. Also the $m$ is unknown at this point and will be determined later.

From Equations (35)-(36), we obtain

$$
\frac{\mathrm{d} U(\xi)}{\mathrm{d} \xi}=A m\left(\tanh ^{m-1} \xi-\tanh ^{m+1} \xi\right)
$$

and

$$
\frac{\mathrm{d}^{2} U(\xi)}{\mathrm{d} \xi^{2}}=A m\left\{(m-1) \tanh ^{m-2} \xi-2 m \tanh ^{m} \xi+(m+1) \tanh ^{m+2} \xi\right\},
$$

and

$$
U^{3}(\xi)=A^{3} \tanh ^{3 m} \xi .
$$

Substituting Equations (35)-(39) into Equation (19), gives

$$
\begin{aligned}
& -\lambda A \tanh ^{m} \xi+\frac{k d}{3} A^{3} \tanh ^{3 m} \xi \\
& +A m k\left(e k^{2}+f p^{2}+g q^{2}\right)\left\{(m-1) \tanh ^{m-2} \xi\right. \\
& \left.-2 m \tanh ^{m} \xi+(m+1) \tanh ^{m+2} \xi\right\}=0 .
\end{aligned}
$$

Now, from Equation (40), equating the exponents of $\tanh ^{3 m} \xi$ and $\tanh ^{m+2} \xi$ gives,

$$
3 m=m+2,
$$

which yields

$$
m=1 \text {. }
$$

Setting the coefficients of $\tanh ^{3 m} \xi$ and $\tanh ^{m+2} \xi$ terms in Equation (40) 
to zero, we have

$$
\frac{k d}{3} A^{3}+A k m(m+1)\left(e k^{2}+f p^{2}+g q^{2}\right)=0
$$

then, we get

$$
A= \pm \sqrt{-\frac{6\left(e k^{2}+f p^{2}+g q^{2}\right)}{d} .}
$$

Again, from Equation (40) setting the coefficients of $\tanh ^{m} \xi$ terms to zero,

$$
-\lambda A-2 m^{2} A k\left(e k^{2}+f p^{2}+g q^{2}\right)=0,
$$

and from Equation (45) we have

$$
\lambda=-2 k\left(e k^{2}+f p^{2}+g q^{2}\right) .
$$

Equation (46) prompts the constraint

$$
d\left(e k^{2}+f p^{2}+g q^{2}\right)<0 .
$$

Thus finally, the dark soliton solution for the $(3+1)$ dimensional space-time fractional mKDV-ZK equation is given by:

$$
\begin{gathered}
u_{3}(x, y, z, t)=\sqrt{-\frac{6\left(e k^{2}+f p^{2}+g q^{2}\right)}{d}} \tanh \left(k x+p y+q z+\frac{2 k\left(e k^{2}+f p^{2}+g q^{2}\right)}{\Gamma(1+\alpha)}\right), \\
u_{4}(x, y, z, t)=-\sqrt{-\frac{6\left(e k^{2}+f p^{2}+g q^{2}\right)}{d}} \tanh \left(k x+p y+q z+\frac{2 k\left(e k^{2}+f p^{2}+g q^{2}\right)}{\Gamma(1+\alpha)}\right)
\end{gathered}
$$

\section{Conclusion}

In this article, we have got the new solutions for the $(3+1)$ dimensional spacetime fractional mKDV-ZK equation by using the method of undetermined coefficients. Up to now, we could not find that these solutions were reported in other papers. In order to solve many systems of nonlinear fractional partial differential equations in mathematical and physical sciences, such as, the space-time fractional $\mathrm{mBBM}$ equation, the time fractional $\mathrm{mKDV}$ equation, the nonlinear fractional Zoomeron equation and so on, we can use the method of undetermined coefficients recommended herein would be general to a certain extent.

\section{Acknowledgements}

This work is in part supported by the Natural Science Foundation of China (Grant Nos. 11271008, 61072147).

\section{References}

[1] Baskonus, H.M. and Bulut, H. (2015) On the Numerical Solutions of Some Fractional Ordinary Differential Equations by Fractional Adams-Bashforth-Moulton Method. Open Mathematics, 13, 547-556. https://doi.org/10.1515/math-2015-0052

[2] Bulut, H., Belgacem, F.B.M. and Baskonus, H.M. (2015) Some New Analytical Solutions for the Nonlinear Time-Fractional KdV-Burgers-Kuramoto Equation. Advances in Mathematics and Statistical Sciences, 118-129. 
[3] Baskonus, H.M., Mekkaoui, T., Hammouch, Z. and Bulut, H. (2015) Active Control of a Chaotic Fractional Order Economic System. Entropy, 17, 5771-5783. https://doi.org/10.3390/e17085771

[4] Cao, J. and Xu, C. (2013) A High Order Schema for the Numerical Solution of the Fractional Ordinary Differential Equations. Journal of Computational Physics, 238, 154-168.

[5] Hammouch, Z. and Mekkaoui, T. (2012) Travelling-Wave Solutions for Some Fractional Partial Differential Equation by Means of Generalized Trigonometry Functions. International Journal of Applied Mathematical Research, 1, 206-212.

[6] Guo, S., Mei, L., Li, Y. and Sun, Y. (2012) The Improved Fractional Sub-Equation Method and Its Applications to the Space-Time Fractional Differential Equations in Fluid Mechanics. Physics Letters A, 376, 407-411.

[7] Gurefe, Y., Sonmezoglu, A. and Misirli, E. (2011) Application of the Trial Equation Method for Solving Some Nonlinear Evolution Equations Arising in Mathematical Physics. Pramana-Journal of Physics, 77, 1023-1029.

https://doi.org/10.1007/s12043-011-0201-5

[8] Taghizadeh, N., Mirzazadeh, M. and Samiei Paghaleh, A. (2012) The First Integral Method to Nonlinear Partial Differential Equations. Applications and Applied Mathematics: An International Journal, 7, 117-132.

[9] Sun, H.G., Zhang, Y., Chen, W. and Reeves, D.M. (2014) Use of a Variable-Index Fractional-Derivative Model to Capture Transient Dispersion in Heterogenerous Media. Journal of Contaminant Hydrology, 157, 47-58.

[10] Fan, E.G. (2000) Extended Tanh-Function Method and Its Applications to Nonlinear Equations. Physics Letters A, 277, 212-218.

[11] Wang, M., Li, X. and Zhang, J. (2008) The (G'/G)-Expansion Method and Travelling Wave Solutions of Nonlinear Evolution Equations in Mathematical Physics. Physics Letters A, 372, 417-423.

[12] Zhang, J., Wei, X. and Lu, Y. (2008) A Generalized (G'/G)-Expansion Method and Its Applications Evolution Equations in Mathematical Physics. Physics Letters A, 372, 3653-3658.

[13] Biswas, A. and Zerrad, E. (2010) Solitary Wave Solution of the Zakharov-Kuznetsov Equation in Plasmas with Power Law Onolinearity. Nonlinear Analysis B, 11, 32723274.

[14] Biswas, A. and Zerrad, E. (2009) 1-Soliton Solution of the Zakharov-Kuznetsov Equation with Dual-Power Law Nonlinerity. Communications in Nonlinear Science and Numerical Simulation, 14, 3574-3577.

[15] Ebadi, G., Mojaver, A., Milovic, D., Johnson, S. and Biswas, A. (2012) Solitons and Other Solutions to the Quantum Zakharov-Kuznetsov Equation. Astrophysics and Space Science, 341, 507-513.

[16] Oldham, K.B. and Spaniner, J. (1974) The Fractional Calculus. Academic Press, New York.

[17] Jumarie, G. (2012) An Approach to Differential Geometry of Fractional Order via Modified Riemann-Liouville Derivative. Acta Mathematica Sinica, English Series, 28, 1741. https://doi.org/10.1007/s10114-012-0507-3

[18] Jumarie, G. (2006) Modified Riemann-Liouville Derivative and Fractional Taylor Series of Nondifferentiable Functions Further Results. Computers \& Mathematics with Applications, 51, 1367.

[19] He, J.H., Elagan, S.K. and Li, Z.B. (2012) Geometrical Explanation of the Fractional Complex Transform and Derivative Chain Rule for Fractional Calculus. Physics Le- 
tters $A, 376,257$.

[20] Ibrahim, R.W. (2012) Fractional Complex Transforms for Fractional Differential Equations. Advances in Difference Equations, 2012, 192. https://doi.org/10.1186/1687-1847-2012-192

[21] Kilbas, A.A., Srivastava, H.M. and Trujillo, J.J. (2006) Theory and Application of Fractional Differential Equations. Elsevier, New York.

[22] Matebese, B.T., Adem, A.R., Khalique, C.M. and Biswas, A. (2011) Solutions to Zakharov-Kuznetsov Equation with Power Law Nonlinearity in (1+3)-Dimensions. Physics of Wave Phenomena, 19, 148-154. https://doi.org/10.3103/S1541308X11020117

[23] Lslam, M.H., Khan, K., Ali Akbar, M. and Salam, M.A. (2014) Exact Traveling Wave Solutions of Modified Kdv-Zakharov-Kuznetsov Equation and Viscous Burger Equation. Springer Plus, 3, 105. https://doi.org/10.1186/2193-1801-3-105

[24] Guner, O., Bekir, A., Moraru, L. and Biswas, A. (2015) Bright and Dark Soliton Solutions of the Generalized Zakharov-Kuznetsov-Benjamin-Bona-Mahoney Nonlinear Evolution Equation, Dark Soliton and Periodic Wave Solutions of Nonlinear Evolution Equations. Proceedings of the Romanian Academy, 16, 422-429.

[25] Bakodah, H.O., Al Qarni, A.A., Banaja, M.A., Zhou, Q., Moshokoa, S.P. and Biswas, A. (2017) Bright and Dark Thirring Optical Solitons with Improved Adomian Decomposition Scheme. Optik, 130, 1115-1123.

Submit or recommend next manuscript to SCIRP and we will provide best service for you:

Accepting pre-submission inquiries through Email, Facebook, LinkedIn, Twitter, etc. A wide selection of journals (inclusive of 9 subjects, more than 200 journals)

Providing 24-hour high-quality service

User-friendly online submission system

Fair and swift peer-review system

Efficient typesetting and proofreading procedure

Display of the result of downloads and visits, as well as the number of cited articles

Maximum dissemination of your research work

Submit your manuscript at: http://papersubmission.scirp.org/

Or contact jamp@scirp.org 